\title{
Reliability and Lifetime Distribution Analysis of Belt Drive Systems considering Time-Dependent Stiffness of Belts
}

\author{
Peng Gao $\mathbb{i D}^{1,2}$ and Liyang Xie $\mathbb{i D}^{3}$ \\ ${ }^{1}$ School of Mechanical Engineering, Liaoning Shihua University, Liaoning 113001, China \\ ${ }^{2}$ School of Mechanical Engineering and Automation, Zhejiang Sci-Tech University, Hangzhou 310018, China \\ ${ }^{3}$ School of Mechanical Engineering and Automation, Northeastern University, Liaoning 110819, China \\ Correspondence should be addressed to Peng Gao; gaopeng@lnpu.edu.cn
}

Received 2 September 2017; Revised 15 January 2018; Accepted 26 February 2018; Published 4 April 2018

Academic Editor: Didier Rémond

Copyright (c) 2018 Peng Gao and Liyang Xie. This is an open access article distributed under the Creative Commons Attribution License, which permits unrestricted use, distribution, and reproduction in any medium, provided the original work is properly cited.

\begin{abstract}
Time-dependent reliability models, failure rate models, and lifetime distribution models of belt drive systems are developed in this paper, which take geometric parameters, material parameters, and motion parameters as the input and consider the dynamic properties of the belt drive systems. Most of the reliability models of belt drive systems are static models. The proposed models can take into account the time-dependent statistical properties of dynamic stress process. Moreover, the stiffness degradation is considered in the established models, whose influences on reliability indices are analyzed in case study. The results show that stiffness degradation has significant impacts on reliability, failure rate, and lifetime distribution. In addition, sensitivity models of reliability with respect to input parameters are constructed, which can be used for the guidance of safe design of belt drive systems.
\end{abstract}

\section{Introduction}

As an important form of mechanical drive, belt drive systems have been widely used in various mechanical products including precision machineries and control systems [1-3]. For many mechanical systems, the working performance and service lifetime are determined by the belt drive systems. In practice, despite the advantages of high transmission efficiency and low cost, belt drive systems are always encountered with the problems of vibration and fatigue, which substantially reduces the operational lifetime of belt drive systems [4]. Hence, it is vital to carry out reliability analysis of belt drive systems to enhance the quality of mechanical systems.

In the last few decades, a great deal of innovative work has been concentrated on the dynamic analysis of belt drive systems. For instance, $\mathrm{Hu}$ et al. established a threedimensional dynamic finite element model to consider the pulley misalignment associated with its effects on dynamic characteristics of belt drive systems [5]. Besides, three types of misalignment were presented in the literature. In order to achieve the velocity regulation of belts by correcting the feedback control, Matsuda et al. developed a control approach to reduce the velocity-fluctuation resulting from belt thickness-variation [6]. Eliseev and Vetyukov simplified the belt drive systems by a nonlinear string model and revealed the effects of chosen strain measure on the parameters of steady operation [7]. Ding analyzed the steady-state responses of a belt drive system with a one-way clutch [8]. The nonlinear differential equations in the models were solved by using the Galerkin truncation method. Li and Chen modeled the belts as strings and analyzed coupled vibration of belt drive systems by means of the modal method [9].

In general, the main difficulties in dynamic analysis of belt drive systems lie in the mathematical description of belts and the coupling between the motion of belts and that of pulleys. Although some analytical methods for dynamic analysis of belt drive systems have been provided by simplifying the transmission belts as strings or a series of beams, many geometric parameters and material parameters cannot be reflected in these models. Furthermore, excessive simplification could cause computational error. Besides, the results are limited in deterministic dynamic analysis. It is difficult to consider the randomness in the material parameters, 
structural parameters, and forces of belt drive systems in the existing models, which limits the dynamic reliability analysis of belt drive systems. In current reliability models of belt drive systems, the system fatigue failure is analyzed by comparing the static strength of belts and the tension in the belts. Correspondingly, the system reliability is derived by calculating the possibility that the static strength is larger than the tension. However, the derivation of the tension lacks further explanation and the reliability calculated is static. As a matter of fact, the tension is essentially dynamic and related to parameters of material, structure, and loads. Furthermore, degradation of material parameters always takes place, such as the strength degradation or the stiffness degradation. The statistical properties of tension and those of the corresponding stress under the tension are time-dependent. In this case, the assumptions of ergodic and stationary process about the dynamic tension and stress cannot be satisfied, which limits the application of conventional rain flow counting (RFC) method in the fatigue lifetime distribution analysis. Therefore, it is necessary to develop dynamic reliability models of belt drive systems considering their dynamic characteristics with the material parameters, structural parameters, and forces as input, which are seldom reported but conducive to random lifetime analysis of belt drive systems. In practice, the driving pulley and the driven pulley seldom fail to work before the failure of belts. However, the dynamic stress in the belts is directly decided by the motion of the pulleys. Therefore, in this paper, dynamic reliability models and random lifetime models of belt drive systems are established based on the system dynamic properties.

This paper is structured as follows. Dynamic stress in belts associated with the method for obtaining its distribution is derived in Section 2. The time-dependent reliability models and lifetime distribution models of belt drive systems considering time-dependent stiffness of belts are developed in Section 3. Sensitivity models are provided in Section 4. Section 5 presents a case study and the conclusions are summarized in Section 6.

\section{Random Dynamic Stress in Belts}

In this section, all the deterministic responses serve as a series of statistical samples to obtain the distribution of the random load in the belts for the time-dependent reliability analysis and the random lifetime distribution analysis. Currently, there exists a large amount of literatures on deterministic dynamic response analysis and static reliability analysis of belt drive systems. However, the belt drive systems show obvious dynamic working mechanism. Therefore, it is necessary to establish time-dependent reliability models to evaluate the quality and safety of the systems in their entire life cycle. At present, time-dependent system reliability models are seldom reported. Moreover, fatigue life analysis of the belts is mostly completed under load with constant amplitude. Nevertheless, the dynamic fluctuation and the randomness of load are comprehensively encountered in practice. The joint effects of the randomness in dynamic load, material parameters, and structural parameters on lifetime distributions of belt drive systems are seldom reported. The main purpose of this paper

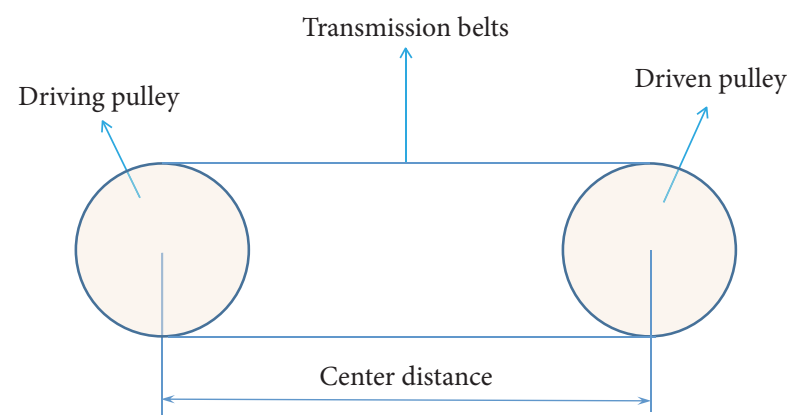

FIGURE 1: Structure of belt drive systems.

is to provide a theoretical basis for time-dependent reliability analysis and the random lifetime distribution analysis.

2.1. Problems in Solution of Dynamic Stress via Analytical Models. Typical structure of a belt drive system can be seen in Figure 1. The driving pulley inputs energy for the operation of the whole system. The belts transfer the input energy via the friction between the pulleys and the belts. To investigate the dynamic characteristics of the belt drive systems, the motion of the driving pulley and the driven pulleys can be expressed as follows:

$$
M_{1} \ddot{\psi}_{1}+C_{1} \dot{\psi}_{1}+K_{1} \psi_{1}=F_{1} \text {, }
$$

where $\psi_{1}=\left[\begin{array}{llll}x_{1} & y_{1} & x_{2} & y_{2}\end{array}\right]$ are the generalized coordinates of the centroids of the driving pulley and the driven pulleys. $F_{1}$ includes the normal pressure and the friction from the belts and the forces caused by mass eccentricity.

When the boundary conditions of (1) are given, the motion of the endpoints of the belt spans can be obtained, which can be taken as the boundary conditions of the string models and the beam models for the motion of the belt spans. The dynamic stress and the fatigue failure are closely correlated with the vibration of belts in the transverse direction. Thus, the investigation in the vibration in the transverse direction accounts for the majority of the research in the vibration of belts. Generally, the string model for the motion in the transverse direction of the belt spans can be expressed as follows [9]:

$$
\rho \frac{\partial^{2} y}{\partial t^{2}}+2 \rho V \frac{\partial^{2} y}{\partial x \partial t}+\left(\rho v^{2}-F\right) \frac{\partial^{2} y}{\partial x^{2}}=0,
$$

where $\rho$ is the mass of belts per unit length, $V$ is the velocity of the belts, and $F$ is the initial tension in the belts. In addition, the beam model for the motion in the transverse direction of the belt spans can be calculated as follows [10]:

$$
\rho \frac{\partial^{2} y}{\partial t^{2}}-2 \rho V \frac{\partial^{2} y}{\partial x \partial t}+\left(\rho V^{2}-F\right) \frac{\partial^{2} y}{\partial x^{2}}+E I \frac{\partial^{4} y}{\partial x^{4}}=0 .
$$

From (2) and (3), it can be learned that it is difficult to acquire an accurate response of the belts. Moreover, the simplification in the modeling of normal pressure and friction, the simplification in the solution of the motion of belt spans, and the coupling between the transverse vibration 
and the longitudinal vibration make it hard to obtain accurate dynamic tension and dynamic stress. Besides, owing to the existence of crack as well as its propagation, the average or equivalent stiffness of belts presents a time-dependent variation, which make it more difficult to derive the motion equation of the belt drive systems and obtain the dynamic stress. Furthermore, current work for dynamic analysis of belt drive systems is mainly focused on deterministic response analysis. In the reliability evaluation of belt drive systems, the randomness of both the input parameters and the timedependent parameters in the motion equations has to be take into account. The methods for time-dependent reliability analysis of belt drive systems are seldom reported.

2.2. Random Dynamic Tension and Stress in Belts via Simulations. In this paper, the multibody program ADAMS is adopted to consider the system dynamic properties and derive the dynamic tension in the belts for system reliability assessment. For different components in a belt drive system, the system dynamic equation can be derived by the Lagrange equation as follows [11-13]:

$$
\frac{d}{d t}\left(\frac{\partial Q}{\partial \dot{\xi}}\right)-\frac{\partial Q}{\partial \xi}+\frac{\partial U}{\partial \dot{\xi}}+\left[\frac{\partial \Omega}{\partial \xi}\right]^{T} \zeta-L=0, \quad \Omega=0,
$$

where $\Omega$ is the constraint equations, $\zeta$ is the Lagrangian multiplier, and $\xi$ is the generalized coordinates. Besides, $U$ stands for the energy dissipation function, $L$ represents the generalized force, and $Q$ is the difference between the system kinetic energy and the system potential energy. Then, the motion equations of a system can be expressed by [11]

$$
\begin{aligned}
& M \ddot{\xi}+\dot{M} \dot{\xi}-\frac{1}{2}\left[\frac{\partial M}{\partial \xi} \dot{\xi}\right]+K \xi+D \dot{\xi}+\left[\frac{\partial \Omega}{\partial \xi}\right]^{T} \zeta-L \\
& \quad=0
\end{aligned}
$$

where $M, K$, and $D$ are the mass matrix, the stiffness matrix, and the damping matrix, respectively. In the simulation model, the input power is represented by the time-dependent velocity of driving pulley. The force and motion are transferred through the pressure and friction between the belts and the pulleys as shown in Figure 2 with the friction coefficient expressed as follows [14]:

$$
\begin{aligned}
& \Xi \\
& = \begin{cases}a_{1} & \left|v_{1}\right|>v_{2} \\
\frac{a_{1}+a_{2}}{2}+\frac{1}{2}\left[\left(a_{2}-a_{1}\right) \cos \left(\pi \frac{\left|v_{1}\right|-v_{2}}{v_{3}-v_{2}}\right)\right] & v_{2} \leq v_{1} \leq v_{3} \\
a_{2} * \sin \left(\frac{\pi\left|v_{1}\right|}{2 v_{2}}\right) & v_{1}<v_{2},\end{cases}
\end{aligned}
$$

where $v_{1}, v_{2}$, and $v_{3}$ are the relative velocity between the pulley and belts, the stick-slip conversion speed, and the static-sliding conversion speed, respectively. $a_{1}$ and $a_{2}$ are the sliding friction coefficient and the static friction coefficient. The pulleys are modeled as rigid bodies. In order to consider the flexibility of the belts, the belts are always divided into a series of small segments that are connected with flexible elements as shown in Figure 2. These flexible elements can be regard as a generalized elastic force vector denoted by $\vec{F}=\left[F_{x}, F_{y}, F_{z}, T_{x}, T_{y}, T_{z}\right] . F_{\text {. }}$ and $T$. represent a force and a moment in a certain direction, respectively [15-17]. It should be noted that when the belts are discretized into a series of rigid bodies with equal length, periodic excitation could be incorporated in the system. This problem can be resolved by introducing some randomness in the definition of rigid elements length.

In practice, the vibration of the belts always comes from the external motion input into the belt drive systems, which significantly influences the fatigue lifetime of the belts drive systems. To describe this external motion, besides the rotation, a periodic translational velocity is applied to the centroid of the driving pulley as shown in Figure 2.

By using the established model, the dynamic tension and stress can be obtained. However, the results are deterministic rather than random. To express the randomness in the dynamic stress, the response surface methodology is adopted in this paper and the assumptions about the motion of the belts are listed as follows.

(1) The operational duration $T$ of the belts is divided into a series of time interval $t_{i}(i=1,2, \ldots, n)$

(2) The statistical characteristics of the dynamic stress change due to the stiffness degradation, which occurs gradually and can be mathematically expressed by the probability density function (PDF) $f\left(w_{i}\right)(i=$ $1,2, \ldots, n)$ in each time interval.

In the situation where the external motion is random, the dynamic stress also presents its random characteristics. Provided that the motion parameters are denoted by $\psi=$ $\left[\begin{array}{llll}\psi_{1} & \psi_{2} & \cdots & \psi_{n_{2}}\end{array}\right]$, the stress $w$ can be obtained via the response surface methodology as follows:

$$
\begin{aligned}
w= & c_{0}+\sum_{i_{1}=1}^{n_{2}} c_{i_{1}} x_{i_{1}}+\sum_{i_{2}=1}^{n_{2}} c_{i_{2} i_{2}} x_{i_{2}}^{2} \\
& +\sum_{i_{3}=1}^{n_{2}-1} \sum_{j_{1}=i_{3}+1}^{n_{2}} c_{i_{3} j_{1}} x_{i_{3}} x_{j_{1}}+\cdots
\end{aligned}
$$

In the case of sufficient samples, the coefficients in (7) can be acquired via the least square method.

Due to the time-dependent statistical characteristics of the dynamic stress, the interference of dynamic stress and dynamic strength in each time interval has to be considered, which is different from the conventional RFC method under ergodic stationary stress. Provided that the statistics of the instant distribution of the dynamic stress are carried out in each time interval, the following data are needed:

$$
A_{1}=\left[\begin{array}{cccc}
a_{1}^{(1)} & a_{2}^{(1)} & \cdots & a_{n_{1}}^{(1)} \\
a_{1}^{(2)} & a_{2}^{(2)} & \cdots & a_{n_{1}}^{(2)} \\
\cdots & \ldots & \ldots & \ldots \\
a_{1}^{(n)} & a_{1}^{(n)} & \cdots & a_{n_{1}}^{(n)}
\end{array}\right],
$$




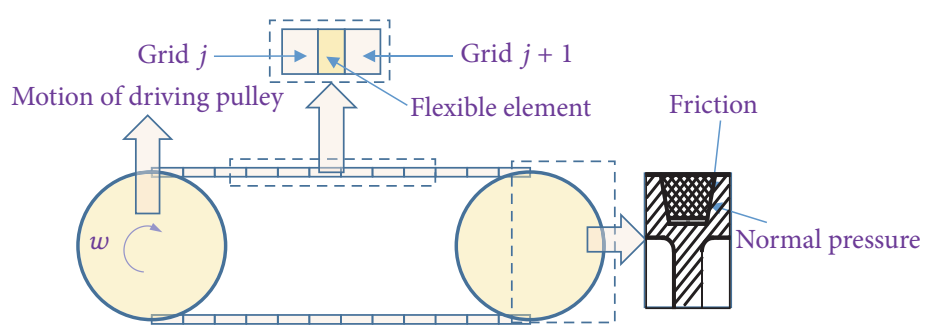

FIgURE 2: Multibody dynamics model of belt drive systems.

where $a_{j}^{(i)}$ is the $j$ th statistical stress in the $i$ th time interval. The number of the statistical data is equal to $i * j$, which is enormous. To improve the statistics, the following equation in material mechanics is employed:

$$
w(t)=\frac{F_{1} E}{K(t) \Delta l} .
$$

$K(t)$ is the time-dependent equivalent stiffness of belts. As a matter of fact, the stiffness degradation takes place due to the propagation of internal cracks of belts. The propagation of internal cracks decreases the effective cross-sectional area, which leads to an increase in both the stress and the deformation of belts. Hence, in this paper, an increasing equivalent coefficient $\beta(t)$ of belts is adopted to describe the propagation of internal cracks and the corresponding stiffness degradation. Denote an initial deterministic dynamic stress by $w_{1}$. The ratio of $w_{1}$ to the dynamic stress in the $i$ th time interval $w_{i}$ originating from $w_{1}$ is expressed as follows:

$$
\beta(t)=\frac{w_{1}}{w_{i}}
$$

Then, the PDF of the dynamic stress in the $i$ th time interval $f_{i}\left(w_{i}\right)$ can be calculated by

$$
f_{i}\left(w_{i}\right)=\beta(t) f_{1}\left(\beta(t) w_{i}\right),
$$

where $f_{1}\left(w_{1}\right)$ is the PDF of initial dynamic stress. Then the work in stress statistics can be substantially reduced.

\section{Reliability and Random Lifetime Models considering Time-Dependent Stiffness}

At present, the literature on reliability of belt drive systems is mostly based on the stress-strength interference model (SSID) which is a static reliability model and considers the interference between the stress and the fatigue limit. The time-dependent reliability models of the belt drive systems based on dynamic properties are seldom reported. The static model cannot take into account the influences of the structure and material parameters on the system reliability. Besides, the SSID neglect the dynamic stress application process where the statistical characteristics of stress vary with time, which could result in a large computational error in system reliability.
According to the S-N Curve theory, the relationship between the stress $w$ and the lifetime $N$ under $w$ can be mathematically expressed as follows:

$$
N w^{m}=C,
$$

where $m$ and $C$ are material parameters. The fatigue limit indicates the stress $w_{0}$ under which the lifetime of the component is larger than a specified lifetime $N_{0}$. In practice, $N_{0}$ is always set to be $10^{6}$. Then the reliability of the belts can be calculated by using the SSID as follows:

$$
R=\int_{0}^{\infty} f_{w}(w) \int_{w}^{\infty} f_{w_{0}}\left(w_{0}\right) d w_{0} d w
$$

From (13), it can be seen that the static reliability model does not consider the working mechanism of belts. To take into account the degradation of strength with time, the equivalent strength in each time interval $N_{1},\left(N_{2}-N_{1}\right), \ldots,\left(N_{n}-N_{n-1}\right)$ can be given by

$$
r_{i}=\frac{C}{N_{i}} \quad(i=1,2, \ldots, n) .
$$

Then, consider the randomness of the material parameter of $C$; the instant reliability in the $i$ th time interval can be written as

$$
R_{i}=\int_{0}^{\infty} f_{C}(C) \int_{-\infty}^{C / N_{i}} f_{w_{i}}\left(w_{i}\right) d w_{i} d C
$$

where $f_{w_{i}}\left(w_{i}\right)$ is the PDF of stress in the $i$ th time interval, which can be acquired by means of the method proposed in Section 2.2. It should be noted that the instant reliability is not the time-dependent reliability of belts and cannot be used to derive the random lifetime distribution of belts. The instant reliability only takes the interference of stress and strength at a specified time instant into consideration and neglects the stress application process.

The reliability of belts within $N_{n}$ can be expressed as follows:

$$
R\left(N_{n}\right)=\int_{0}^{\infty} f_{C}(C) \prod_{i=1}^{n} \int_{-\infty}^{C / N_{i}} f_{w_{i}}\left(w_{i}\right) d w_{i} d C
$$


The corresponding failure rate of the belts is given by

$$
\begin{aligned}
h\left(N_{n}\right) & =\frac{F\left(N_{n+1}\right)-F\left(N_{n}\right)}{R\left(N_{n}\right)}=\frac{R\left(N_{n}\right)-R\left(N_{n+1}\right)}{R\left(N_{n}\right)} \\
& =\frac{\int_{0}^{\infty} f_{C}(C) \prod_{i=1}^{n} \int_{-\infty}^{C / N_{i}} f_{w_{i}}\left(w_{i}\right) d w_{i}\left(1-\int_{-\infty}^{C / N_{n+1}} f_{w_{n+1}}\left(w_{n+1}\right) d w_{n+1}\right) d C}{\int_{0}^{\infty} f_{C}(C) \prod_{i=1}^{n} \int_{-\infty}^{C / N_{i}} f_{w_{i}}\left(w_{i}\right) d w_{i} d C} .
\end{aligned}
$$

According to the relationship between reliability and lifetime distribution, the PDF of the system lifetime can be expressed as follows:

$$
f\left(N_{n}\right)=\frac{\partial}{\partial t}\left[1-R\left(N_{n}\right)\right]=\frac{\int_{0}^{\infty} f_{C}(C) \prod_{i=1}^{n} \int_{-\infty}^{C / N_{i}} f_{w_{i}}\left(w_{i}\right) d w_{i}\left(1-\int_{-\infty}^{C / N_{n+1}} f_{w_{n+1}}\left(w_{n+1}\right) d w_{n+1}\right) d C}{N_{n+1}-N_{n}} .
$$

It should be noted that (16), (17), and (18) consider the stiffness degradation. When the stiffness degradation is neglected, the reliability, failure rate, and lifetime PDF of the belt drive systems can be rewritten as follows.

System reliability:

$$
R_{1}\left(N_{n}\right)=\int_{0}^{\infty} f_{C}(C) \prod_{i=1}^{n} \int_{-\infty}^{C / N_{i}} f_{w}(w) d w d C
$$

System failure rate:

$$
\begin{aligned}
& h_{1}\left(N_{n}\right) \\
& =\frac{\int_{0}^{\infty} f_{C}(C) \prod_{i=1}^{n} \int_{-\infty}^{C / N_{i}} f_{w}(w) d w\left(1-\int_{-\infty}^{C / N_{n+1}} f_{w}(w) d w\right) d C}{\int_{0}^{\infty} f_{C}(C) \prod_{i=1}^{n} \int_{-\infty}^{C / N_{i}} f_{w}(w) d w d C} .
\end{aligned}
$$

System lifetime PDF:

$$
\begin{aligned}
& f_{1}\left(N_{n}\right) \\
& =\frac{\int_{0}^{\infty} f_{C}(C) \prod_{i=1}^{n} \int_{-\infty}^{C / N_{i}} f_{w}(w) d w\left(1-\int_{-\infty}^{C / N_{n+1}} f_{w}(w) d w\right) d C}{N_{n+1}-N_{n}} .
\end{aligned}
$$

\section{Sensitivity Models}

From the analysis in Section 3, it can be learned that the motion, the pulleys, and the stiffness degradation have significant impacts on the system reliability, failure rate, and the system lifetime distribution. In addition, these impacts change with time. To quantify these impacts and analyze their variation with time, the sensitivity models with respect to motion parameters of driving pulleys and $\beta(t)$ are provided as follows.
(1) Sensitivity of system reliability with respect to mean value of motion parameters $\mu(\psi)=\left[\mu\left(\psi_{1}\right) \quad \mu\left(\psi_{2}\right) \cdots\right.$ $\left.\mu\left(\psi_{n_{2}}\right)\right]$ :

$$
\begin{array}{r}
k_{1, j_{3}}=\frac{\partial\left[\int_{0}^{\infty} f_{C}(C) \prod_{i=1}^{n} \int_{-\infty}^{C / N_{i}} f_{w_{i}}\left(w_{i}\right) d w_{i} d C\right]}{\partial \mu\left(\psi_{j_{3}}\right)} \\
\left(j_{3}=1,2, \ldots, n_{2}\right) .
\end{array}
$$

(2) Sensitivity of system reliability with respect to standard deviation of motion parameters $\sigma(\psi)=$ $\left[\begin{array}{llll}\sigma\left(\psi_{1}\right) & \sigma\left(\psi_{2}\right) & \cdots & \sigma\left(\psi_{n_{2}}\right)\end{array}\right]:$

$$
\begin{array}{r}
k_{2, j_{4}}=\frac{\partial\left[\int_{0}^{\infty} f_{C}(C) \prod_{i=1}^{n} \int_{-\infty}^{C / N_{i}} f_{w_{i}}\left(w_{i}\right) d w_{i} d C\right]}{\partial \mu\left(\psi_{j_{4}}\right)} \\
\left(j_{4}=1,2, \ldots, n_{2}\right) .
\end{array}
$$

(3) Sensitivity of system reliability with respect to $\beta(t)$ :

$$
k_{3}=\frac{\partial\left[\int_{0}^{\infty} f_{C}(C) \prod_{i=1}^{n} \int_{-\infty}^{C / N_{i}} f_{w_{i}}\left(w_{i}\right) d w_{i} d C\right]}{\partial \beta(t)} .
$$

\section{Case Study}

Consider a belt drive system composed of a driving pulley, a driven pulley, and transmission belts with the geometric parameters and the material parameters listed in Table 1. Besides rotation, the motion of driving pulley in the vertical direction is expressed by $v(t)=\Delta \sin (20 \pi t+\phi)$. The amplitude $\Delta$ follows the normal distribution with the mean value of $12 \mathrm{~mm}$ and the standard deviation of $2 \mathrm{~mm} . \beta\left(N_{i}\right)$ is given by

$$
\beta\left(N_{i}\right)=\frac{1}{1+(i-1) / 8000} .
$$




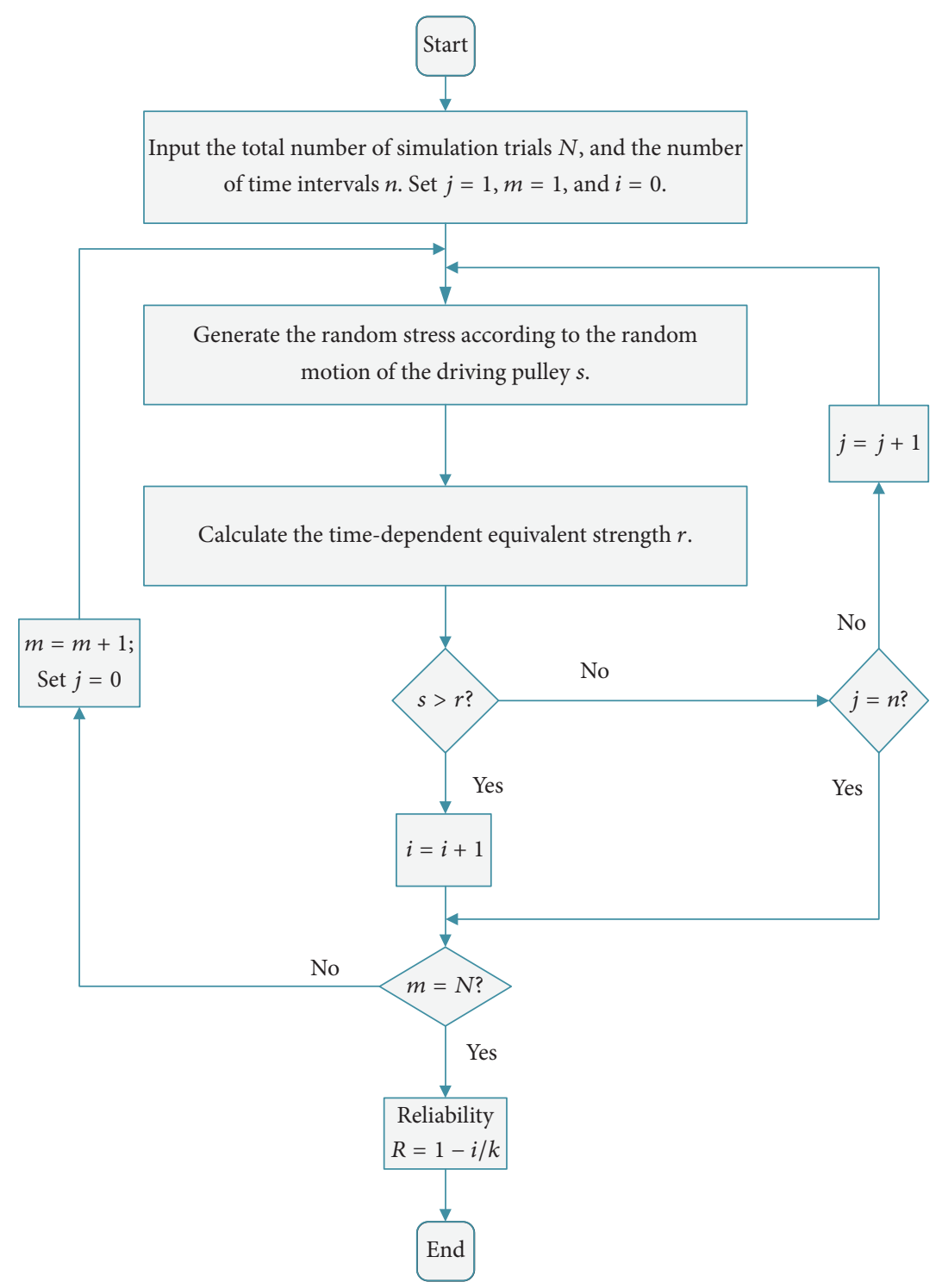

Figure 3: Monte Carlo simulation.

In practice, the operational duration is divided into a series of subintervals with the stiffness in each subinterval remaining constant. Then, the stiffness degradation law can be obtained by using the curve fitting techniques with the stiffness tested in each subinterval $[18,19]$. The reliability, failure rate, and lifetime PDF of the belts with stiffness degradation and those without stiffness degradation are shown in Figures 4-6.

When considering the randomness of material parameters and stiffness degradation, it is unaffordable or impractical to perform the physical experiment of belts with a large amount of belt samples to validate the proposed models. To overcome this problem, the method of integrating both the simulation via ADAMS and the Monte Carlo simulations is used in this paper. In fact, due to the difficulty in obtaining a large number of experimental samples, Monte Carlo simulations (MCS), rather than physical experiment, have been widely used for reliability validation in existing literatures. In the MCS, the randomness in load, material parameters are considered and the working process is consistent with the actual working process. The purpose of the MCS is to verify the validity and effectiveness of the theoretical framework of time-dependent reliability estimation proposed in this paper. The Mote Carlo simulation with the flowchart in Figure 3 is shown in Figure 4. Besides, the instant reliability with stiffness degradation and that without stiffness degradation are shown in Figure 7. In addition, the sensitivities of reliability with respect to the statistical parameters of $\Delta$ and $\beta\left(N_{i}\right)$ are shown in Figures 8-10.

From Figures $4-7$, it can be learned that stiffness degradation has great influences on the system reliability, the system failure rate, and the system lifetime PDF. In general, the stiffness degradation decreases the system reliability and increases the failure rate of belt drive systems. The difference between the reliability considering stiffness degradation and that without stiffness degradation considered increases with the usage time of the belt drive systems. Moreover, the results 


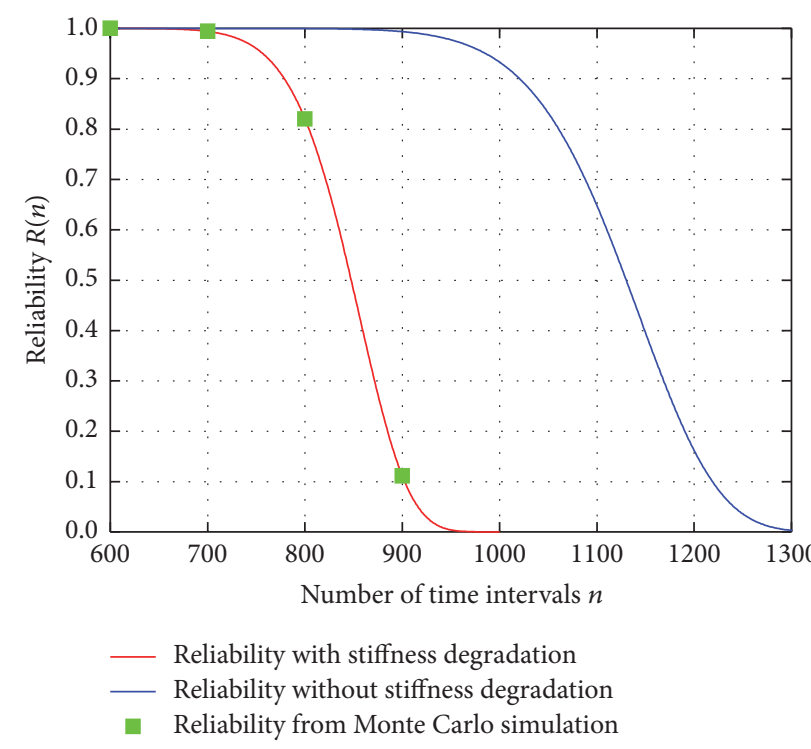

FIGURE 4: Reliability from proposed models and reliability from Monte Carlo simulations.

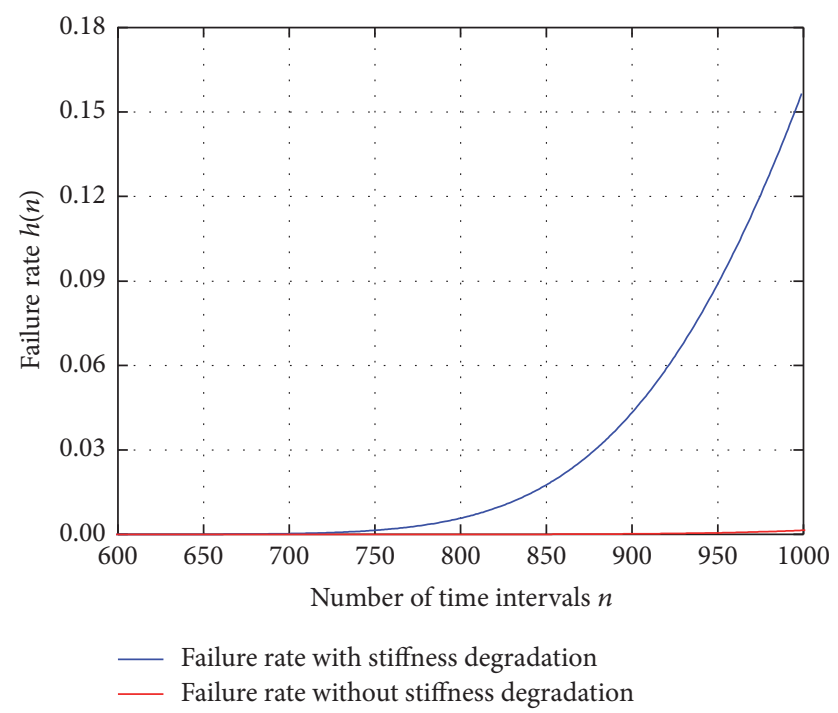

FIGURE 5: Failure rate of belt drive systems.

TABLE 1: Geometric parameters and the material parameters.

\begin{tabular}{lcc}
\hline Parameters & Value & Units \\
\hline Density of pulley & $7.8 * 10^{3}$ & $\mathrm{~kg} / \mathrm{m}^{3}$ \\
Density of belts & $5.3 * 10^{3}$ & $\mathrm{~kg} / \mathrm{m}^{3}$ \\
Velocity of belts & 25 & $\mathrm{~m} / \mathrm{s}$ \\
Center distance of pulleys & 500 & $\mathrm{~mm}$ \\
Diameters of pulleys & 100 & $\mathrm{~mm}$ \\
Cross-sectional area of belts & 30 & $\mathrm{~mm}^{2}$ \\
Number of time intervals & 1500 & \\
Number of stress applications per time interval & 1000 & \\
$m$ & 3.2 & \\
$C$ & $10^{10}$ & $\mathrm{MPa}^{2}$ \\
\hline
\end{tabular}

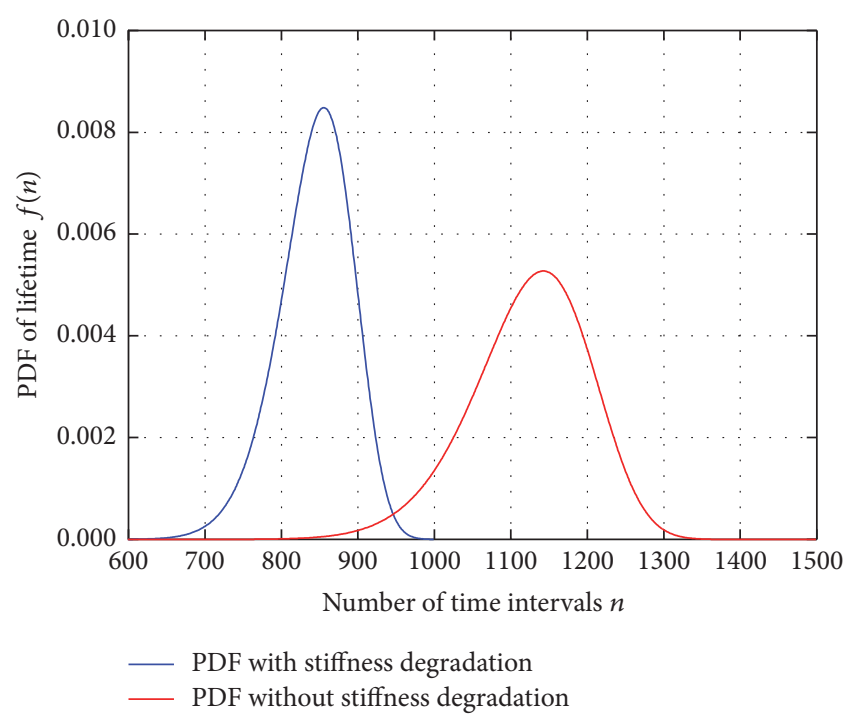

FIgURE 6: Lifetime PDF of belt drive systems.

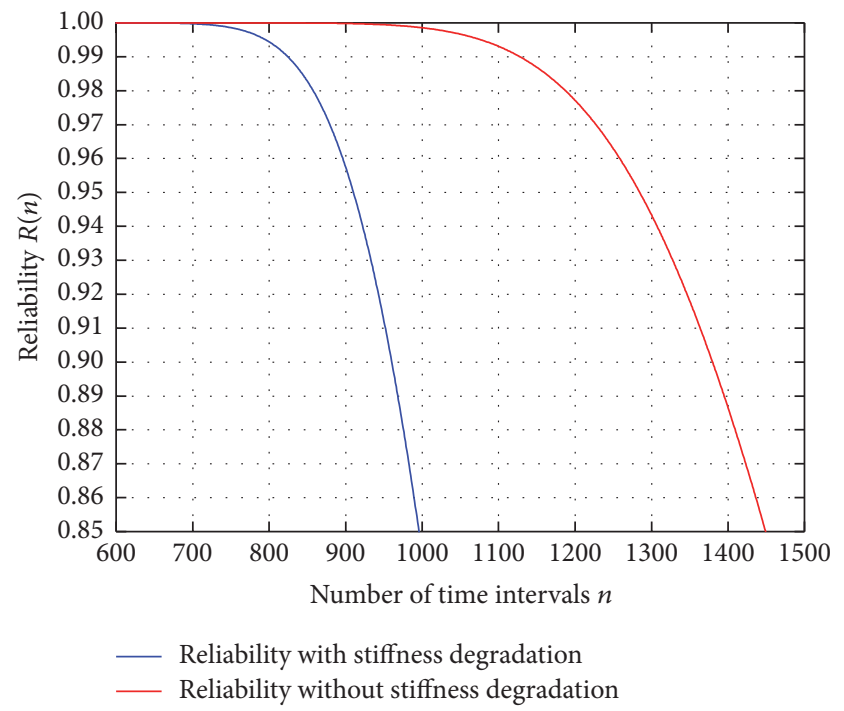

FIGURE 7: Instant reliability of belt drive systems.

from the Monte Carlo simulation validate the effectiveness of the proposed models. Besides, the stiffness degradation lowers the mean value of the system lifetime and lessens the dispersion of the lifetime distribution. In addition, the instant reliability is also effected by the stiffness degradation. Furthermore, the instant reliability is significantly higher than the system reliability, because the instant reliability only considers the interference between the dynamic stress and the strength at a specified moment. The stress application history is not reflected in the instant reliability. Therefore, when calculating reliability of belt drive systems, the usage of instant reliability could cause large computational error.

From Figures 4-7, it can be seen that the time-dependent reliability is sensitive to the mean value and standard deviation of $\Delta$ in the stage where reliability significantly decreases. Besides, the reliability is more sensitive to the standard devia- 


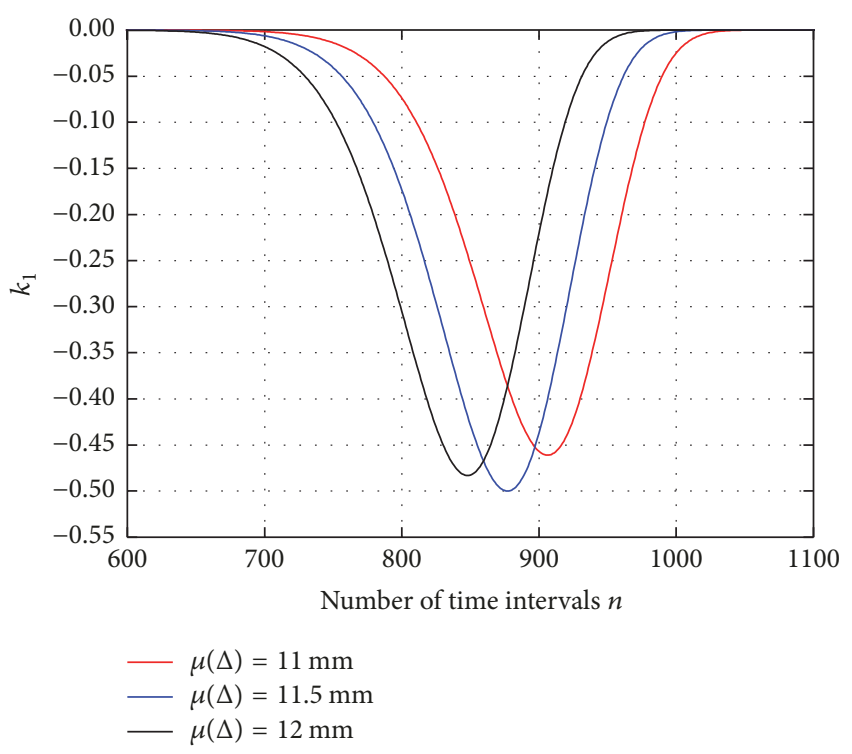

Figure 8: Sensitivity with respect to mean value of $\Delta$.

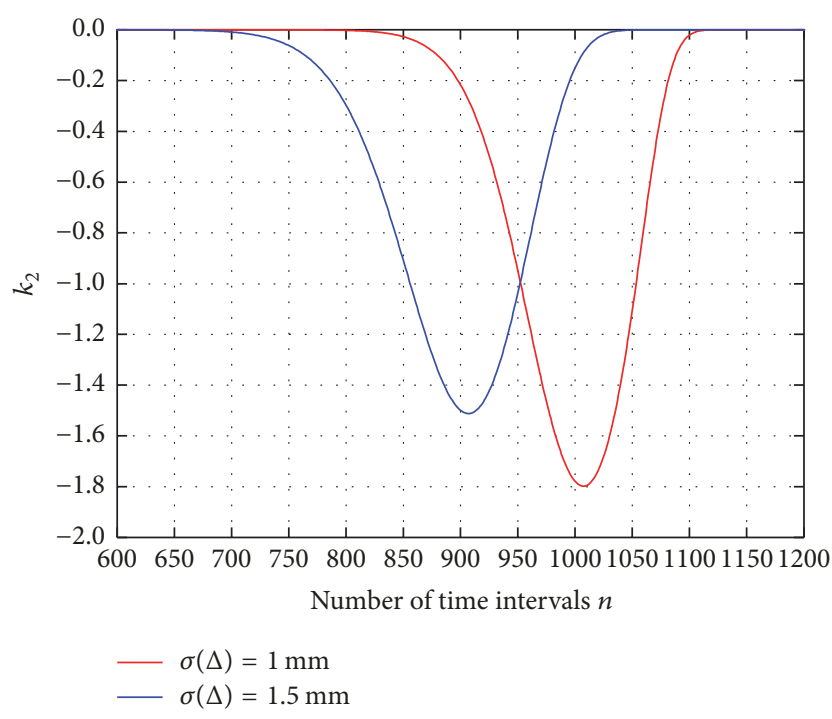

FIGURE 9: Sensitivity with respect to standard deviation of $\Delta$ in the case where mean value of $\Delta$ is $12 \mathrm{~mm}$.

tion of $\Delta$ than the mean value of $\Delta$. Furthermore, the peak value in the sensitive interval comes earlier as the mean value of $\Delta$ or the standard deviation of $\Delta$ increases. In addition, the system reliability is also sensitive to $\beta\left(N_{i}\right)$ with the peak value of sensitivities lying in the position where reliability significantly decreases.

\section{Conclusions}

The failure of the belt drive systems occurs due to the interaction between the belts and the pulleys. Time-dependent reliability analysis based on multibody dynamics models can take this interaction into consideration. The proposed time-dependent reliability models are developed based on the dynamic properties of the belt drive systems with the

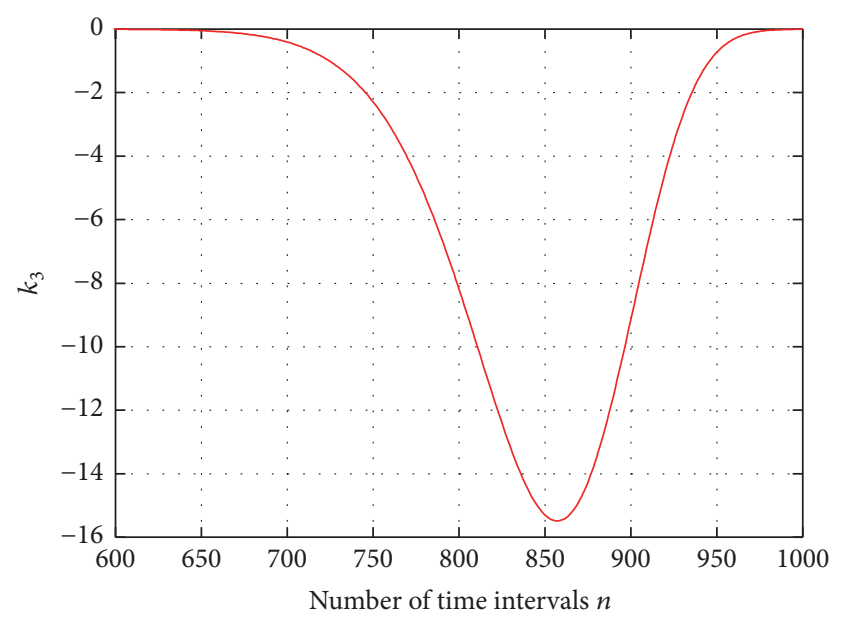

FIgURE 10: Sensitivity with respect to $\beta\left(N_{i}\right)$.

geometric parameters, the material parameters, and the motion parameters as the input of the models. Moreover, the established models take into account the stiffness degradation and can consider the time-dependent statistical properties of dynamic stress, which cannot be accomplished by using the conventional RFC method that is limited to the condition of ergodic stationary stress process. The influences of stiffness degradation on time-dependent reliability, failure rate, and lifetime distribution are analyzed by using the proposed models. The results show that stiffness degradation has considerable impacts on these reliability indices. In addition, the system reliability presents different sensitivities to different input parameters in different usage stage, which should be paid attention to in the design of the belt drive systems.

\section{Conflicts of Interest}

The authors declare that they have no conflicts of interest.

\section{Acknowledgments}

This work is supported by National Natural Science Foundation of China (Grant no. 51505207), Program for Liaoning Innovative Talents in University (Grant no. LR2017070), Liaoning Provincial Natural Science Foundation of China (Grant no. 2015020152), and Open Foundation of Zhejiang Provincial Top Key Academic Discipline of Mechanical Engineering (Grant no. ZSTUME02A01).

\section{References}

[1] J. Wu, S. Yan, J. Li, and Y. Gu, "Mechanism reliability of bistable compliant mechanisms considering degradation and uncertainties: modeling and evaluation method," Applied Mathematical Modelling, vol. 40, no. 23-24, pp. 10377-10388, 2016.

[2] R. S. Beikmann, N. C. Perkins, and A. G. Ulsoy, "Design and analysis of automotive serpentine belt drive systems for steady state performance," Journal of Mechanical Design, vol. 119, no. 2, pp. 162-168, 1997.

[3] T. C. Firbank, "Mechanics of the belt drive," International Journal of Mechanical Sciences, vol. 12, no. 12, pp. 1053-1063, 1970. 
[4] V. S. Utkin, "Reliability of a belt-drive shaft characterized by fuzzy and random basic parameters," Russian Engineering Research, vol. 30, no. 7, pp. 668-670, 2010.

[5] J. Hu, J. Chen, S. Sundararaman, and K. Chandrashekhara, "Finite element analysis of V-ribbed belt/pulley system with pulley misalignment using a neural-network-based material model," Neural Computing and Applications, vol. 18, no. 8, pp. 927-938, 2009.

[6] H. Matsuda, T. Andoh, and H. Koide, "Color registration error reduction method compensating the influence of belt thickness variation in electrophotographic process," Microsystem Technologies, vol. 13, no. 8-10, pp. 1425-1430, 2007.

[7] V. Eliseev and Y. Vetyukov, "Effects of deformation in the dynamics of belt drive," Acta Mechanica, vol. 223, no. 8, pp. 1657-1667, 2012.

[8] H. Ding, "Steady-state responses of a belt-drive dynamical system under dual excitations," Acta Mechanica Sinica, vol. 32, no. 1, pp. 156-169, 2016.

[9] X.-J. Li and L.-Q. Chen, "Modal analysis of coupled vibration of belt drive systems," Applied Mathematics and Mechanics, vol. 29, no. 1, pp. 9-13, 2008.

[10] S. K. Kim and J. M. Lee, "Analysis of the non-linear vibration characteristics of a belt-driven system," Journal of Sound and Vibration, vol. 223, no. 5, pp. 723-740, 1999.

[11] L. Chen, Mechanical system dynamics analysis and ADAMS Application Tutorial, Tsinghua University Press, Tsinghua, China, 2005.

[12] Z. Qin, D. Cui, S. Yan, and F. Chu, "Hysteresis modeling of clamp band joint with macro-slip," Mechanical Systems and Signal Processing, vol. 66, 2015.

[13] Z. Y. Qin, S. Z. Yan, and F. L. Chu, "Influence of clamp band joint on dynamic behavior of launching system in ascent flight," Proceedings of the Institution of Mechanical Engineers, Part G: Journal of Aerospace Engineering, vol. 228, no. 1, pp. 97-114, 2014.

[14] J. Wu, S. Yan, and M. J. Zuo, "Evaluating the reliability of multibody mechanisms: A method considering the uncertainties of dynamic performance," Reliability Engineering \& System Safety, vol. 149, pp. 96-106, 2016.

[15] R. Zhang, L. Y. Zhang, and C. Zhao, "Simulation analysis of belt conveyors based on ADAMS," Hoisting Machinery, vol. 7, pp. 33-36, 2017.

[16] S. S. Hao, Y. X. Li, and X. T. Liu, "Establishment method of belt conveyor solid simulation model based on adams," Coal Mine Machinery, vol. 29, no. 2, pp. 178-180, 2008.

[17] S. S. Hao, X. T. Liu, and X. L. Liu, "Study on dynamic characteristics of belt conveyor during startup process based on ADAMS," Mining Machinery, vol. 40, no. 6, pp. 60-63, 2012.

[18] R. Mikalauskas and V. Volkovas, "Analysis of the dynamics of a defective V-belt and diagnostic possibilities," Proceedings of the Institution of Mechanical Engineers, Part I: Journal of Systems and Control Engineering, vol. 220, no. 2, pp. 145-153, 2006.

[19] R. Mikalauskas and V. Volkovas, "Modeling of defects of flexible elements of bel drives," in Proceedings of the XVI IMEKO World Congress, pp. 233-238, September 2000. 


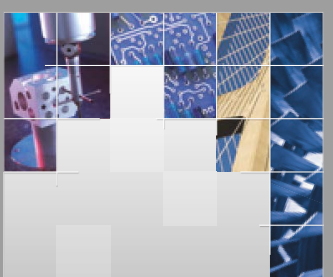

\section{Enfincering}
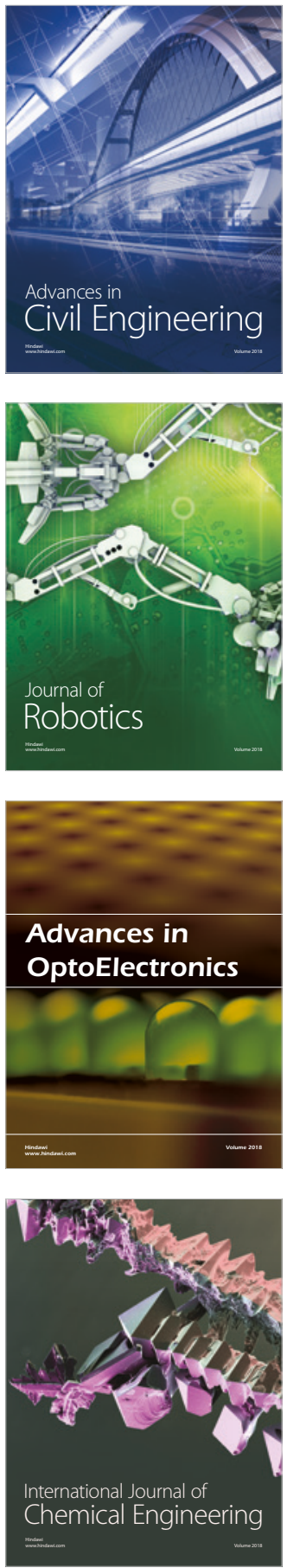

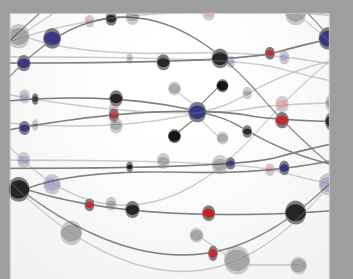

\section{Rotating \\ Machinery}

The Scientific World Journal

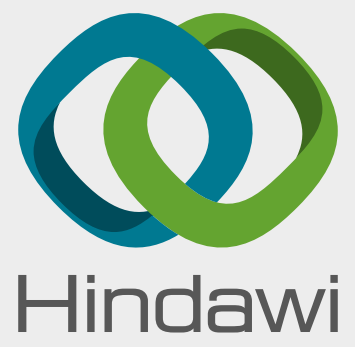

Submit your manuscripts at

www.hindawi.com
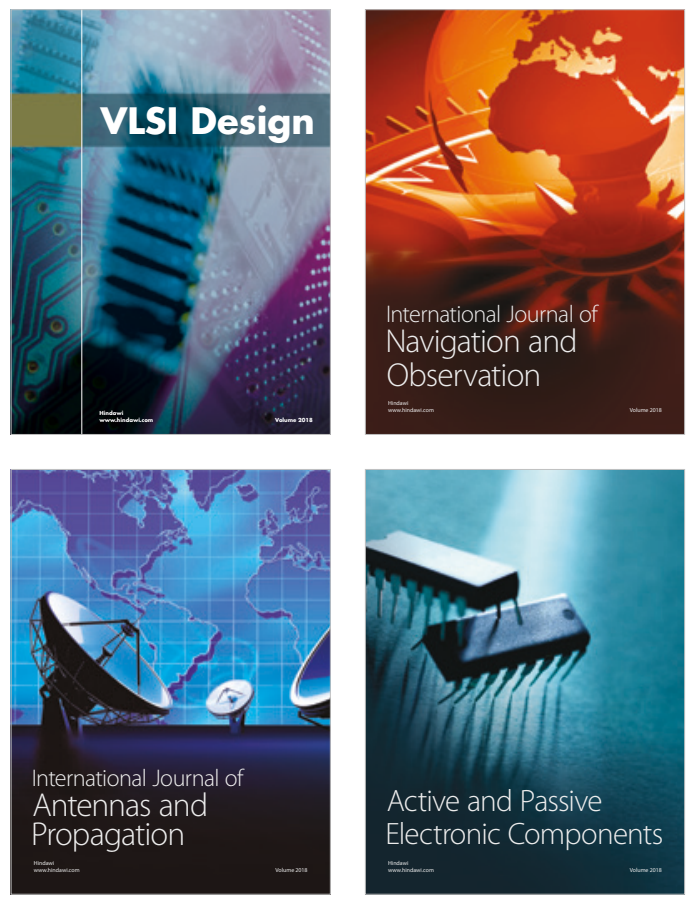
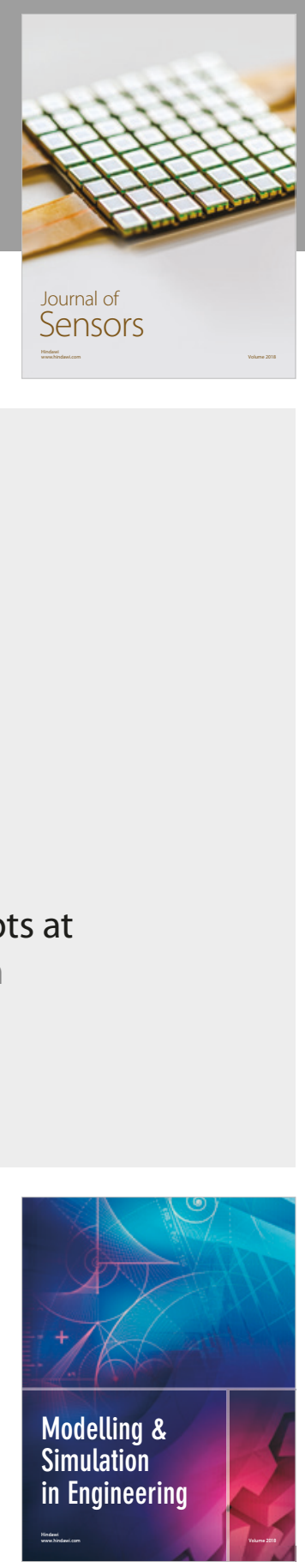

\section{Advances \\ Multimedia}
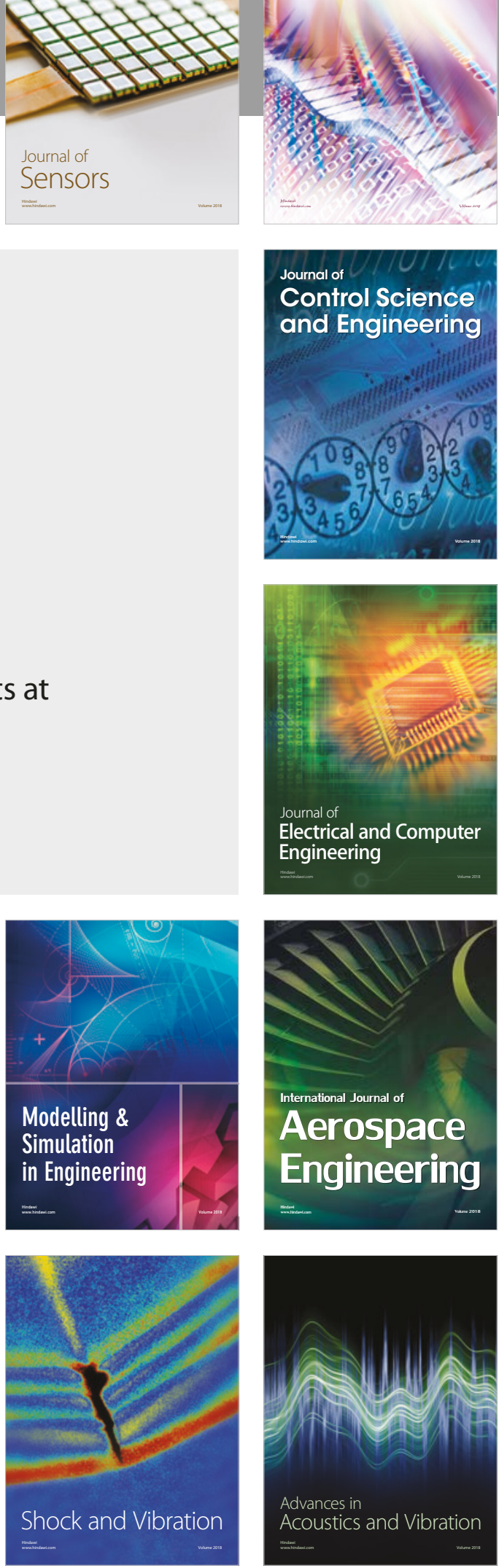